\title{
Will recording of lung function fluctuation open the door to internet-guided treatment of asthma?
}

\author{
Maciej Kupczyk, ${ }^{*}$ Sven-Erik Dahlén
}

Asthma is a heterogeneous and variable chronic disease. Although it is very common, affecting more than $5 \%$ of adults and $10 \%$ of children in most parts of the world, asthma remains a great challenge both with respect to proper diagnosis and the means proactively to adjust treatment in a disease that is characterised by periods of worsening and remissions. The diagnostic difficulties are mirrored in the complex definition of asthma which comprises four domains: airway obstruction, symptoms, airway inflammation and airway hyper-responsiveness. ${ }^{1}$ None of these domains alone is sufficient for diagnosis but together they describe the clinical and pathophysiological face of the syndrome of asthma. Nowadays it is widely accepted that the main goal of asthma treatment is to reach and maintain good control of the disease and, in particular, to prevent and limit periods of flare-ups. In order to improve treatment there is a great need to provide the doctor with a better objective understanding of the level of disease control over time during the patient's daily activities rather than only judging from history and examinations at a visit to the clinic. There is also a desire to find ways to give patients more effective support for self-management, as asthma is a disease where environmental triggers and lifestyle factors play a significant part. Taken together, asthma is the perfect case for internet-governed personalised medicine. It remains, however, to implement this vision because sufficiently sensitive and specific measures have not been established.

The Unit for Experimental Asthma and Allergy Research, The National Institute of Environmental Medicine and The Centre for Allergy Research at Karolinska Institutet, Stockholm, Sweden

*Post doctoral fellow supported by the Bernhard Osher Initiative for Research on Severe Asthma

Correspondence to Professor Sven-Erik Dahlén, The Unit for Experimental Asthma and Allergy Research, The National Institute of Environmental Medicine, P O Box 287, Karolinska Institutet, Stockolm SE-117 77, Sweden; sven-erik.dahlen@ki.se
In previous editions of the Global Initiative for Asthma (GINA) recommendations for asthma treatment, strong emphasis was placed on lung function measurement. Recording of forced expiratory volume in $1 \mathrm{~s}\left(\mathrm{FEV}_{1}\right)$ and peak expiratory flow (PEF) provides an objective day-to-day measure of airway obstruction and is one of the most common physiological variables reported in clinical practice and asthma trials. ${ }^{2}$ However, there is an inconsistent relationship between lung function measurements and symptoms or exacerbation frequency as patient-centred outcome measures, ${ }^{3}$ and the use of daily PEF recordings has generally not lived up to its promise. In fact, in clinical studies there is quite a weak correlation between clinical variables, physiological biomarkers, lung function, number of exacerbations and level of asthma treatment. ${ }^{2}$ In real-life practice the level of asthma control is still most often judged by a 'global physician assessment'. Although this approach seems to be intuitively valid, it is very difficult to standardise and useless to help the patient with feedback for selfmanagement. More recent recommendations of GINA $^{1}$ list many variables (daytime symptoms, limitation of activities, nocturnal awakenings, need for rescue treatment, lung function and the number of exacerbations) that should be taken into account when defining disease control. Inherent to this proposal is that observation over longer time periods is necessary for proper assessment of asthma control. Use of day and night symptoms and rescue medication should be based on at least 1 week of observation, and the number of exacerbations needs to be recorded during 1 year of follow-up. ${ }^{1}$

Accordingly, recordings of variation in lung function over a longer period of time may provide more valid information than a single static measurement of lung function. Previous simplified approaches introduced a reference level for the patient's 'personal best' (recorded over at least 2 weeks of optimal treatment and good control of the disease) that could be used to calculate 'in-between day variability' in lung function. The research group led by Frey in Basel, Switzerland has, however, in recent years taken the dynamic analysis of lung function data much further by the introduction of advanced biostatistics and mathematical modelling. For example, they found that the time series of PEF show long-range correlations that change significantly with disease severity and can be used to predict exacerbations and unstable airway function. ${ }^{4}$

In this issue of Thorax Thamrin et al ${ }^{5}$ report on associations between fluctuations in lung function and asthma control in two asthma cohorts with different severity of disease. They take advantage of a new and interesting mathematical tool-detrended fluctuation analysis-to evaluate the hypothesis that a past history of fluctuations in lung function provides additional information for the assessment of asthma control. It has been found that $\alpha$, a variable representing the strength of correlation with past lung function and asthma stability, and PEF, both evaluated over 6 months of followup, were significantly associated with the GINA-defined level of control. However, $\alpha$ was related to whether or not exacerbations occurred in mild to moderate asthma whereas PEF seemed to be more predictive of exacerbations in the cohort with severe asthma. The authors conclude that fluctuation analysis may provide further insight into the future risk of an individual patient having exacerbations of the disease. Incorporation into asthma selfmanagement plans of such measurements with greater probability to predict worsening may thus help to optimise the level of asthma control and therefore improve the quality of healthcare.

The findings in the present report confirm and extend the message in previous studies from this group-namely, that time series analysis of PEF provides more predictive information. ${ }^{4}$ Furthermore, in another paper by Thamrin et al ${ }^{6}$ it has even been suggested that calculation of individual conditional probabilities based on PEF data from only 64 days of observation makes it possible to determine the future risk of clinically-defined exacerbations in patients with asthma. The novel method of analysing fluctuations in lung function clearly holds the promise to be an important step towards better phenotyping of asthma at a population level, and has the potential to be applied to personalised medicine at the patient level. It can be hypothesised that the 
quantitative approach proposed by Frey and coworkers may be extended and applied also to fluctuations of clinical symptoms, biomarkers, medication use and other endpoints used to monitor asthma.

Before lung function history indices can be implemented in our everyday clinical practice, more studies are required to improve the understanding of this new tool. For example, can the same measures of lung function history be applied to patients with asthma of different severity? The present study by Thamrin et $a l^{5}$ represents the first approach to this issue and, as mentioned, the data suggest that different indices may be needed in severe asthma than in mild to moderate asthma. However, the present study does not allow us to draw final conclusions on this issue. The studies of mild to moderate and severe asthma were made on two sets of data originating from two very different studies performed about 10 years apart and, as the authors point out, one limitation with the older study was that it did not use electronic diaries. Different inclusion criteria, different settings and a different course of the two trials also make the direct comparison of findings in these two cohorts difficult. There is therefore a need for confirmatory studies in well-phenotyped and coherent cohorts of patients with asthma of different severity and identical study protocols. It would be an added benefit if several biomarkers were determined repeatedly over a long time period in parallel with lung function data, symptoms and medication use. This would allow similar calculations of the history of biomarkers, symptoms and other outcomes, possibly adding information about the pathophysiology of asthma.

Considered together, the study of Thamrin et $a l^{5}$ raises excitement of future improvements both in clinical practice and mechanistic research. It seems that fluctuation analysis of data from handheld electronic spirometers should be a valuable new application to add to smartphones. This would make it possible to assess the value of lung function history measurements in research and also in ordinary clinical follow-up. For research, lung function fluctuation analysis may represent an important new key variable which, when integrated with other clinical and biological (genomic, transcriptomic, proteomic and metabolomic) data, may allow for better classification and phenotyping of asthma by the use of mathematical modelling in a systems biology approach. ${ }^{7}$ Such a better understanding of the pathology of asthmaespecially of severe, poorly-controlled or difficult-to-treat cases ${ }^{8}$-may facilitate development of new diagnostic methods and improve asthma care. Moreover, identification of key nodes in the complex network of inflammatory processes underlying asthma may result in discovery of new targets for effective therapeutic intervention.

Competing interests None
Provenance and peer review Not commissioned; internally peer reviewed.

Published Online First 19 August 2011

Thorax 2011;66:1019-1020.

doi:10.1136/thoraxjnl-2011-200847

\section{REFERENCES}

1. Global Initiative for Asthma. Global Strategy for Asthma Management and Prevention, 2008. http://www.ginasthma.com.

2. Reddel HK, Taylor DR, Bateman ED, et al. An official American Thoracic Society/European Respiratory Society statement: asthma control and exacerbations: standardizing endpoints for clinical asthma trials and clinical practice. Am J Respir Crit Care Med 2009;180:59-99.

3. Bousquet J, Mantzouranis E, Cruz AA, et al. Uniform definition of asthma severity, control and exacerbations: document presented for the World Health Organization Consultation on Severe Asthma. J Allergy Clin Immunol 2010;126:926-38.

4. Frey U, Brodbeck T, Majumdar A, et al. Risk of severe asthma episodes predicted from fluctuation analysis of airway function. Nature

2005; 438:667-70

5. Thamrin C, Nydegger R, Stern G, et al. Association between fluctuations in lung function and asthma control in two populations with differing asthma severity. Thorax 2011;66:1036-42.

6. Thamrin C, Zindel J, Nydegger R, et al. Predicting future risk of asthma exacerbations using individual conditional probabilities. J Allergy Clin Immunol 2011;127:1496-502.

7. Auffray C, Adcock IM, Chung KF, et al. An integrative systems biology approach to understanding pulmonary diseases. Chest 2010;137:1410-16.

8. Anon. The ENFUMOSA cross-sectional European multicentre study of the clinical phenotype of chronic severe asthma. European Network for Understanding Mechanisms of Severe Asthma. Eur Respir J 2003;22:470-7.

\title{
The challenges of quality improvement reports and the urgent need for more of them
}

\author{
Kieran McIntyre, ${ }^{1,2}$ Kaveh G Shojania ${ }^{2,3}$
}

Healthcare quality has received sustained attention since the release of To Err is Human by the US Institute of Medicine in

\footnotetext{
'Division of Respirology, St Michael's Hospital, Toronto, Ontario, Canada; ' ${ }^{2}$ Department of Medicine, University of Toronto, Toronto, Ontario, Canada; ${ }^{3}$ Sunnybrook Health Sciences Centre, the University of Toronto, Centre for Patient Safety, Toronto, Ontario, Canada
}

Correspondence to Dr Kieran McIntyre, Division of Respirology, St. Michaels Hospital, Rm 6-037, Toronto, ON M5B 1W8, Canada; mcintyrek@smh.ca late $1999 .{ }^{1}$ This report captured widespread interest with the oft-quoted estimate that medical errors annually cause 44000-98000 deaths in US hospitals alone. This period also coincided with publication of 'An organisation with a memory', ${ }^{2}$ which described the scale and nature of serious failures in the UK National Health Service.

A widely accepted definition describes quality as the degree to which health services for individuals and populations increase the likelihood of desired health outcomes and are consistent with current professional knowledge. ${ }^{3} 4$ This definition further characterised quality in terms of six dimensions: safety, effectiveness, patient centeredness, timeliness, efficiency and equitability.

Numerous studies document major shortcomings in each of these dimensions across a range of clinical settings. ${ }^{3}$ One illustrative study ${ }^{5}$ showed that only $55 \%$ of Americans with chronic medical conditions received basic aspects of acute, chronic and preventive care. ${ }^{5}$ For example, only $50 \%$ of patients with asthma received chronic inhaled corticosteroids and a similarly low percentage of patients with chronic obstructive pulmonary disease (COPD) received influenza vaccination. These major shortfalls in effective healthcare do not simply reflect access issues, as comparable data from Canada (with universal public healthcare) show that only 